\title{
ACCURACY OF VOLUMETRIC MEASUREMENTS AFTER VIRTUAL RIGHT HEPATECTOMY IN POTENTIAL DONORS UNDERGOING LIVING ADULT LIVER TRANSPLANTATION
}

\author{
Rahul Raj1', Nazar Puthukudiyil Kader2, Srikanth Moorthy33, Sreekumar Karumathil Pullara4, Ramiah Rajesh Kannan', \\ Deepak Remakanthan', Rajasekar Chandrasekarn7, Vinoth Alagappan ${ }^{8}$
}

\begin{abstract}
1 Junior Resident, Department of Radiology, Amrita Institute of Medical Sciences and Research Institute. ${ }^{2}$ Associate Professor, Department of Radiology, Amrita Institute of Medical Sciences and Research Institute. 3 Professor, Department of Radiology, Amrita Institute of Medical Sciences and Research Institute. ${ }^{4}$ Professor, Department of Radiology, Amrita Institute of Medical Sciences and Research Institute. ${ }^{5}$ Associate Professor, Department of Radiology, Amrita Institute of Medical Sciences and Research Institute. ${ }_{6}^{6}$ Senior Resident, Department of Radiology, Amrita Institute of Medical Sciences and Research Institute. ${ }^{7}$ Senior Resident, Department of Radiology, Amrita Institute of Medical Sciences and Research Institute. ${ }^{8}$ Senior Resident, Department of Radiology, Amrita Institute of Medical Sciences and Research Institute.
\end{abstract}

\section{ABSTRACT}

\section{BACKGROUND}

Living related liver transplantation in the adult is a new surgical procedure that allows healthy adults to donate a portion of their liver to compatible recipients.[1-3] This innovative procedure is performed to overcome the shortage of available cadaveric livers. Surgery involves removal of the right lobe of the liver (segments V - VIII) from a healthy donor without endangering the vascular supply or metabolic function of the remaining left lobe. Liver procurement is possible because of its unique bilobar multi-segmental anatomy. In addition, regeneration of the remaining left lobe compensates for the loss of hepatic mass. In this study, we evaluated the accuracy of total and segmental liver volume measurements using multi-detector CT in potential donors undergoing adult right lobe liver transplantation.

\section{MATERIALS AND METHODS}

Between May 2014 and March 2016, 31 patients 25 were females (80.6\%) and 6 were males (19.4\%) with a mean age of 39.8 years; range 22 - 58 years who underwent donor hepatectomy were evaluated. Imaging performed using Siemens Somatom Sensation 64 Slice CT after injection of Omnipaque $(1.5 \mathrm{~mL} / \mathrm{kg})$ at a rate of $5 \mathrm{~mL} / \mathrm{sec}$. Arterial dominant phase images were acquired at 6 seconds, portal dominant phase images were acquired at +20 seconds and delayed phase images were acquired at +30 seconds. Bolus tracking with triggering of scan when the density of aorta was $100 \mathrm{HU}$ is used in our Department. Volumetry done using Terarecon Intuition Edition Software by manual and automated methods.

\section{RESULTS}

Of the 14 donor grafts with middle hepatic vein, volume assessed using software-aided automated volumetry when compared with intraoperative graft volume found to have a correlation coefficient of $r=0.811$ and is statistically significant $p<0.001$. Manual volume estimation using $2.5 \mathrm{~mm}$ slice thickness and $5 \mathrm{~mm}$ slice thickness recorded a correlation coefficient of $\mathrm{r}=0.776$ and $\mathrm{r}=0.754$ respectively in comparison to actual graft volume and they are statistically significant with $\mathrm{p}<0.001$ and $\mathrm{p}<0.002$ respectively. In the second group of 17 donors' grafts without middle hepatic vein, volume assessed with software-aided automated volumetry with intraoperative graft volume estimation recorded a correlation coefficient of $r=0.977$ and is statistically significant $p<0.001$. In the same group, manual volume using $5 \mathrm{~mm}$ slice thickness and $2.5 \mathrm{~mm}$ slice thickness had a correlation coefficient of $\mathrm{r}=0.877$ and $\mathrm{r}=$ 0.865 respectively and they are statistically significant with $\mathrm{p}<0.001$ and $\mathrm{p}<0.001$ respectively.

\section{CONCLUSION}

In our study, CT volumetry is an accurate tool for preoperative liver volume calculation. We found Automated CT volumetry method to have a better correlation with the resected liver volume and thinner slices had a slightly more correlation than thicker slices for manual method when compared with the resected liver volume.

\section{KEYWORDS}

Living Adult Liver Transplantation, Volumetric Assessment.

HOW TO CITE THIS ARTICLE: Raj R, Kader NP, Moorthy S, et al. Accuracy of volumetric measurements after virtual right hepatectomy in potential donors undergoing living adult liver transplantation. J. Evolution Med. Dent. Sci. 2017;6(10):777-780, DOI: $10.14260 /$ Jemds/2017/168

Financial or Other, Competing Interest: None.

Submission 11-01-2017, Peer Review 22-01-2017,

Acceptance 26-01-2017, Published 02-02-2017.

Corresponding Author:

Rahul Raj,

Department of Radiology,

Amrita Institute of Medical Sciences and Research Institute,

Ponekkara, Kochi-682041, Kerala.

E-mail: rahulrajelitegroup@gmail.com

DOI: $10.14260 /$ jemds $/ 2017 / 168$

\section{(c) (i) $\$$}

\section{BACKGROUND}

Liver transplantation in adults is a surgical procedure which involves the replacement of a diseased liver with some or whole of a healthy liver from a compatible donor (1-3). It involves removal of the right lobe of the liver (segment Vsegment VIII) from the healthy donor without compromising on the vascular supply or metabolic function of the remaining part of the donor liver. The segmental anatomy allows surgeons to create grafts of varying size, depending upon the recipient's requirement for liver tissue. Since liver has a 
unique feature of regeneration, loss of hepatic tissue will be compensated. The shortage of available cadaveric donor organs is the major limiting factor in liver transplantation In this study we evaluated the accuracy of total and segmental liver volume measurements using multi-detector CT in potential donors undergoing adult right lobe liver transplantation.

\section{MATERIALS AND METHODS}

\section{This is a Prospective Study}

Cross-sectional validity analysis design done over a period from June 2014 to March 2016. The study was approved by the Institutional Review Board. An informed consent was taken from all patients undergoing imaging. Sample size based on the correlation coefficients of manual to intraoperative volume and automated to intraoperative volume observed in an earlier publication and with $20 \%$ allowable error and $95 \%$ confidence, minimum sample size comes to less than 10 . Here, I included a total of 31 cases in my study. This will give the estimated $20 \%$ allowable error and $99 \%$ confidence. Inclusion criteria include all prospective liver donors for patients undergoing liver transplantation and exclusion criteria are patients with fatty liver, focal liver lesions, insufficient liver volumetry and complex vascular anatomy.

\section{Imaging}

Imaging performed using Siemens Somatom Sensation 64 Slice CT after injection of Omnipaque $(1.5 \mathrm{~mL} / \mathrm{kg})$ at a rate of $5 \mathrm{~mL} / \mathrm{sec}$. Arterial dominant phase images were acquired at 6 seconds, portal dominant phase images were acquired at +20 seconds and delayed phase images were acquired at +30 seconds. Bolus tracking with triggering of scan when the density of aorta was $100 \mathrm{HU}$ is used in our Department.

Volumetry done using TeraRecon Intuition edition software by manual and automated methods.

\section{Manual Volumetry}

Liver volume is measured by hand-tracing the liver outline on the axial portal venous phase images. Hand-tracing was performed to isolate the liver from surrounding structures of similar attenuation, such as the stomach and spleen. Handtracing was performed with every axial image. To enhance the accuracy in volumetric measurement, large vessels (including the inferior vena cava and extrahepatic portal vein) and major fissures (such as the fissure for the ligamentum teres) are excluded. The plane avoided major vessels traversing between the right and left lobes, immediately to the right of the middle hepatic vein. The circumscribed areas are then multiplied by the CT section thickness to give an approximate volume for each liver section. The volume of all sections were summed to give total and lobar volume of liver. Manual volumetry was done with $5 \mathrm{~mm}$ and $2.5 \mathrm{~mm}$ slice thickness.

\section{Automated Software for Measuring Liver Volume}

Automated volumetric scheme was used to yield liver volumes. We applied the scheme to venous phase images to maximise the intensity difference between the liver parenchyma and non-liver tissue. First, a program for automated liver extraction was applied to $1 \mathrm{~mm}$ 3D CT images and the liver boundaries were obtained automatically. The software used 3D geodesic-active-contour segmentation coupled with a level set algorithm. The radiologist edited the boundary to enhance the accuracy of volumetry by using a specially designed software tool (restricted region growing technique). Liver volume is automatically calculated by summation of the products of the section thickness and area of segmented liver in each section.

Both manual and automated volumes are compared to intra-operative graft volume, which is taken as the gold standard reference.

\section{Statistical Analysis}

In our study, numerical variables were expressed as mean and standard deviation and categorical variables were expressed as frequency and percentage.

Karl Pearson's correlation coefficient was computed between manual to intraoperative and automated to intraoperative.

\section{RESULTS}

Between May 2014 and March 2016, 31 patients (25 were females $(80.6 \%)$ with a mean age of 39.8 years; range 22 - 58 years who underwent donor hepatectomy were evaluated. of the 14 donor grafts with middle hepatic vein, volume assessed using software aided automated volumetry when compared with intraoperative graft volume found to have a correlation coefficient of $r=0.811$ and is statistically significant $p<0.001$ (Figure 1).

Manual volume estimation using $2.5 \mathrm{~mm}$ slice thickness and $5 \mathrm{~mm}$ slice thickness recorded a correlation coefficient of $r=0.776$ and $r=0.754$ respectively in comparison to actual graft volume and they are statistically significant with $\mathrm{p}<$ 0.001 and $p<0.002$ respectively (Figure 2, Appendix Figure 3). In the second group of 17 donors grafts without middle hepatic vein, volume assessed with software aided automated volumetry with intraoperative graft volume estimation recorded a correlation coefficient of $r=0.977$ and is statistically significant $\mathrm{p}<0.001$ (Appendix Figure 4).

In the same group, manual volume using $5 \mathrm{~mm}$ slice thickness and $2.5 \mathrm{~mm}$ slice thickness had a correlation coefficient of $r=0.877$ and $r=0.865$ respectively and they are statistically significant with $\mathrm{p}<0.001$ and $\mathrm{p}<0.001$ respectively (Appendix Figure 5, 6).

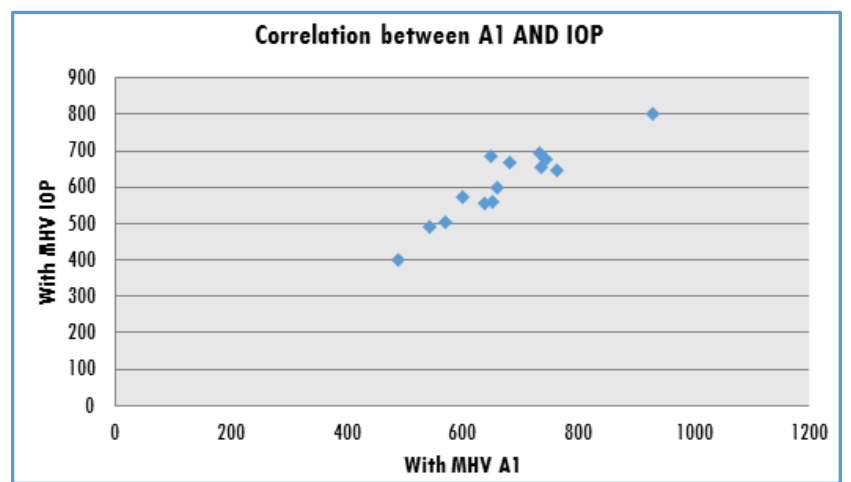

Figure 1. Scatter Plot showing Correlation between Automated Volumetry and Intraoperative Graft Volume 


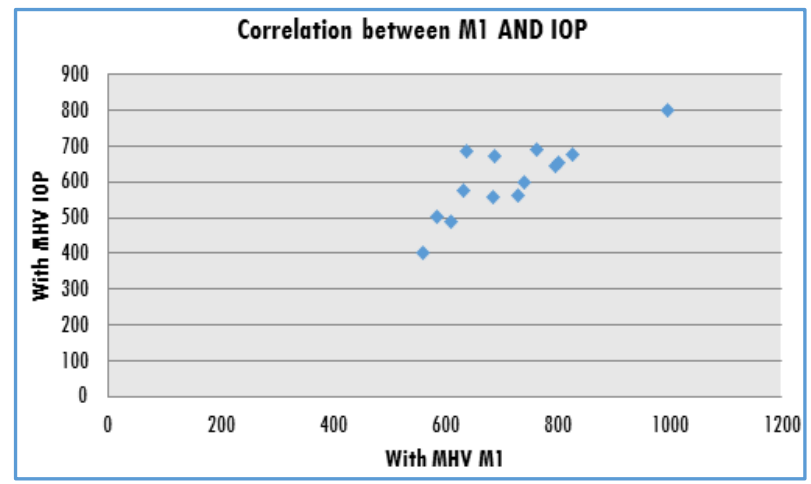

Figure 2. Scatter Plot showing Correlation between Manual Volumetry (M1) and Intraoperative Graft Volume

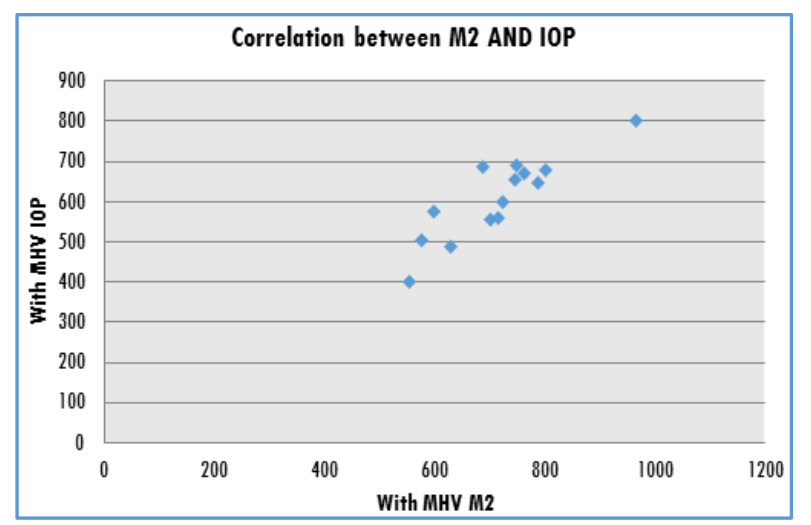

Appendix Figure 1. Scatter Plot showing Correlation between Manual Volumetry (M2) and Intraoperative Graft Volume

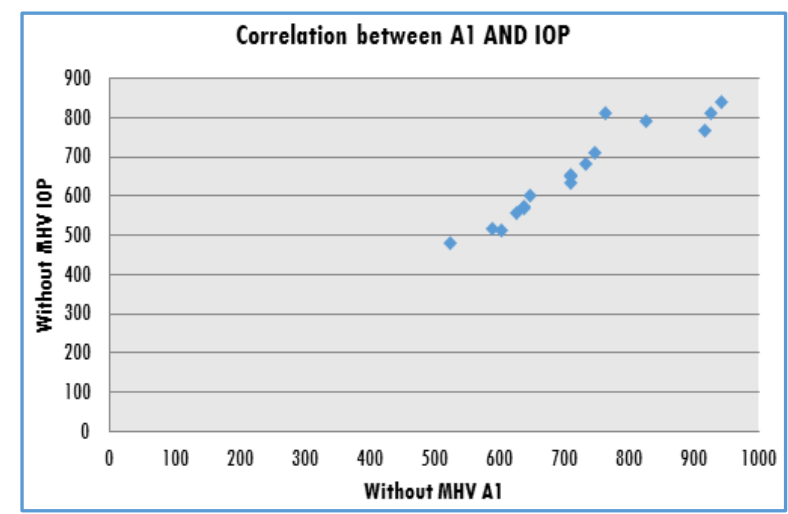

Appendix Figure 2. Scatter Plot showing Correlation between Automated Volumetry and Intraoperative Graft Volume

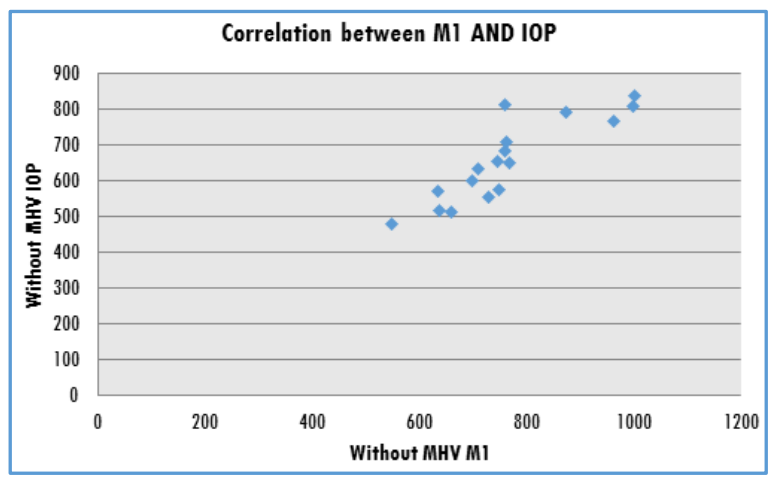

Appendix Figure 3. Scatter Plot showing Correlation between Automated Volumetry and Intraoperative Graft Volume

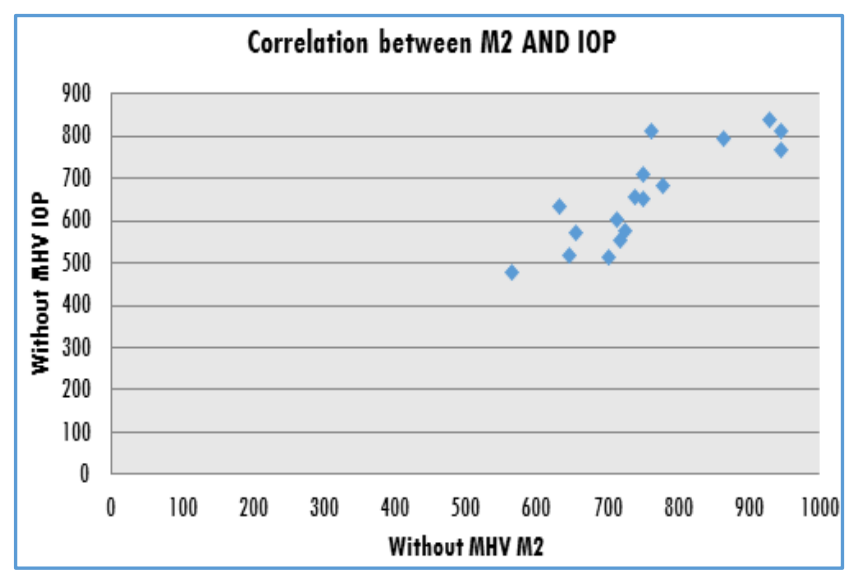

Appendix Figure 4. Scatter Plot showing Correlation between Automated Volumetry and Intraoperative Graft Volume

\section{DISCUSSION}

Living donor liver transplantation in adults is a surgical procedure, which involves the replacement of a diseased liver with part of a healthy liver from a compatible donor. This innovative procedure is performed to overcome the shortage of available cadaveric livers. Surgery involves removal of the right lobe of the liver (segments V - VIII) from a healthy donor without endangering the vascular supply or metabolic function of the remaining left lobe. Liver procurement is possible because of its unique bilobar multi-segmental anatomy, which allows surgeons to create grafts of varying size depending upon the recipient's requirement for liver tissue.

Donor selection is based on total and segmental liver volumes, because graft size has been one of the major factors determining a successful outcome. A small graft may result in malfunction or may not sustain metabolic function in the recipient. A large graft is associated with a risk of graft compression and poor perfusion.(4) In addition, in potential donors it should be made sure that adequate volume of liver is retained to permit metabolic functions during the regeneration process. Therefore, accuracy of total and segmental liver volumes is important to avoid donor-recipient volume mismatch.(5) Each hepatic segment has its own vascular supply and can be resected without affecting the remaining liver parenchyma.[6] Liver is a unique organ in the body, which has a remarkable capacity to regenerate back to $75 \%-95 \%$ of its original mass with complete restoration of its functions in 8 - 15 days after surgery.[7] The most common LDLT technique in adults is right hemihepatectomy, whereby segments V - VIII are harvested, leaving the Middle Hepatic Vein (MHV) with the donor.[8,9]

We conducted this study to know the accuracy of liver volume estimation from CT data using different methods by comparing it with actual graft volume measured intraoperatively. The study sample included 31 donors, 25 female $(80.6 \%)$ and 6 male $(19.4 \%)$ with a mean age of 39 years (age range 22 to 58 years). All these potential liver donors underwent multiphasic contrast enhanced CT of the liver, which was performed as part of preoperative workup.

Graft volume assessed based on requirement of recipient and remnant volume for donor. In this study of 31 donors, intraoperative graft volume of 14 donors were taken along 
with middle hepatic vein, while graft volume of 17 donors were taken without middle hepatic vein to meet the need of necessary volume requirement.

We calculated volume for the two different types of graft procured (right lobe with MHV and right lobe without MHV), using software aided (Terarecon) manual and automated methods of preoperative CT volumetric calculation. Manual volumetry was calculated using both $5 \mathrm{~mm}$ and $2.5 \mathrm{~mm}$ slices to study the effect of slice thickness on volumetry.

Flushed graft weight was measured immediately after donor hepatectomy. It was assumed that the absolute graft weight is the actual graft volume, because the liver has nearly the same density as water and actual volume so measured is taken as the gold standard.

Scatter plots were used to identify the correlation between the right lobe volume measured and the actual graft weight. A linear regression equation was generated for each scatter plot and the correlation coefficient was calculated.

Of the 14 donor grafts with middle hepatic vein, volume assessed using software aided automated volumetry when compared with intraoperative graft volume found to have a correlation coefficient of $r=0.811$ and is statistically significant with $\mathrm{p}<0.001$.

Manual volume estimation using $2.5 \mathrm{~mm}$ slice thickness and $5 \mathrm{~mm}$ slice thickness recorded a correlation coefficient of $r=0.776$ and $r=0.754$ respectively in comparison to actual graft volume and both are statistically significant with $\mathrm{p}<$ 0.001 and $\mathrm{p}<0.002$ respectively.

In the second group of 17 donor grafts without middle hepatic vein, volume assessed with software-aided automated volumetry with intraoperative graft volume estimation recorded a correlation coefficient of $r=0.977$ and is statistically significant with $\mathrm{p}<0.001$.

In the same group, manual volume using $5 \mathrm{~mm}$ slice thickness and $2.5 \mathrm{~mm}$ slice thickness had a correlation coefficient of $r=0.877$ and $r=0.865$ respectively in comparison to actual graft volume and both showed statistically significant value of $p<0.001$ and $p<0.001$ respectively.

From the statistical analysis, it is found that there was an overall good correlation between various methods of preoperative graft liver volume estimation in comparison with actual graft liver volume. However, automated method yielded a better accuracy, as it was done using thinner sections and there was no scope for manual tracing error. Nakayama et al[10] found in their study that both manual and automated CT volumetric methods yielded acceptable measurements when compared with the data obtained from resected liver.

Manual volume estimation using $2.5 \mathrm{~mm}$ slice thickness images had a better correlation in comparison to similar volume estimation using $5 \mathrm{~mm}$ slices with actual graft volume. The mean volume of liver obtained by various methods was overestimated compared to intra-operative graft volume. This is probably due to the fact that after graft liver procurement it is weighed without blood within tissues and blood vessels. Accurately assessing liver volume is critical for the success of living donor liver transplant. Our study validates the manual and automatic volumetric techniques using CT data. Presence of anatomic variation is a challenge for automatic technique. A combination of automation combined machine learning from expert-guided manual methods appears to be the future for this technique.

\section{Study Limitation}

Manual volumetry was done with a single operator. Multioperator is a more real life situation. Intra-operator variation in manual mode was not part of our study. The gold standard in this study is also prone for errors, which were not taken into consideration.

\section{CONCLUSION}

In our study, CT volumetry is an accurate tool for preoperative liver volume calculation. We found Automated CT volumetry method to have a better correlation with the resected liver volume and thinner slices had a slightly more correlation than thicker slices for manual method when compared with the resected liver volume.

\section{REFERENCES}

[1] Broelsch CE, Burdelski M, Rogiers X, et al. Living donor for liver transplantation. Hepatology 1994;20[1 Pt 2]:49S-55S.

[2] Hashikura V, Makuuchi M, Kawasaki S, et al. Successful living-related partial liver transplantation to an adult patient. Lancet 1994;343[8907]:1233-4.

[3] Kawasaki S, Machuuchi M, Maatsunami H, et al. Livingrelated liver transplantation in adults. Ann Surg 1998;227(2):269-74.

[4] Kasai H, Makuuchi M, Kawasaki S, et al. Intraoperative color Doppler ultrasonography for partial-liver transplantation from the living donor in pediatric patients. Transplantation 1992;54[1]:173-5.

[5] Emond JC, Renz JF, Ferrell LD, et al. Functional analysis of grafts from living donors: implications for the treatment of older recipients. Ann Surg 1996;224(4):544-54.

[6] Alonso-Torres A, Fernández-Cuadrado J, Pinilla I, et al. Multidetector CT in the evaluation of potential living donors for liver transplantation. Radiographics 2005;25(4):1017-30.

[7] Michalopoulos GK. Liver regeneration. J Cell Physiol 2007;213[2]:286-300.

[8] Singh AK, Cronin CG, Verma HA, et al. Imaging of preoperative liver transplantation in adults: what radiologists should know. Radiographics 2011;31(4):1017-30.

[9] Tanaka K, Yamada T. Living donor liver transplantation in Japan and Kyoto University: what can we learn? J Hepatol 2005;42[1]:25-8.

[10] Nakayama Y, Li O, Katsurgawa S, Ikeda R, et al. Automated hepatic volumetry for living related liver transplantation at multisection CT. Radiology 2006;240(3):743-8. 